\title{
Blood-based signatures in type 1 diabetes
}

\author{
Susanne M. Cabrera ${ }^{1,2} \cdot$ Yi-Guang Chen ${ }^{1,2} \cdot$ William A. Hagopian $^{3}$. \\ Martin J. Hessner ${ }^{1,2}$
}

Received: 23 September 2015 / Accepted: 18 November 2015/Published online: 23 December 2015

(C) Springer-Verlag Berlin Heidelberg 2015

\begin{abstract}
Type 1 diabetes mellitus is one of the most common chronic diseases in childhood. It develops through autoimmune destruction of the pancreatic beta cells and results in lifelong dependence on exogenous insulin. The pathogenesis of type 1 diabetes involves a complex interplay of genetic and environmental factors and has historically been attributed to aberrant adaptive immunity; however, there is increasing evidence for a role of innate inflammation. Over the past decade new methodologies for the analysis of nucleic acid and protein signals have been applied to type 1 diabetes. These studies are providing a new understanding of type 1 diabetes pathogenesis and have the potential to inform the development of new biomarkers for predicting diabetes onset and monitoring therapeutic interventions. In this review we will focus on bloodbased signatures in type 1 diabetes, with special attention to both direct transcriptomic analyses of whole blood and immunocyte subsets, as well as plasma/serum-induced transcriptional signatures. Attention will also be given to proteomics, microRNA assays and markers of beta cell death. We will also discuss the results of blood-based profiling in type 1 diabetes within the context of the genetic and environmental factors implicated in the natural history of autoimmune diabetes.
\end{abstract}

Martin J. Hessner

mhessner@mcw.edu

1 The Max McGee National Research Center for Juvenile Diabetes, Children's Research Institute of Children's Hospital of Wisconsin, Milwaukee, WI, USA

2 Section of Endocrinology, Department of Pediatrics, The Medical College of Wisconsin, 8701 Watertown Plank Road, Milwaukee, WI 53226, USA

3 Pacific Northwest Diabetes Research Institute, Seattle, WA, USA
Keywords Biomarker $\cdot$ Gene expression profiling $\cdot$ Innate immunity $\cdot$ Microarray $\cdot$ Review $\cdot$ Type 1 diabetes

$\begin{array}{ll}\text { Abbreviations } \\ \text { BB } & \text { BioBreeding } \\ \text { HLA } & \text { Human leukocyte antigen } \\ \text { HRS } & \text { High-risk siblings } \\ \text { I.I.com } & \text { Composite inflammatory index } \\ \text { LRS } & \text { Low-risk siblings } \\ \text { miRNA } & \text { microRNA } \\ \text { PBMC } & \text { Peripheral blood mononuclear cell } \\ \text { PRR } & \text { Pattern recognition receptor } \\ \text { ROT1D } & \text { Recent-onset type 1 diabetes } \\ \text { Treg } & \text { Regulatory T cell } \\ \text { uHC } & \text { Unrelated healthy controls }\end{array}$

\section{Introduction}

Type 1 diabetes is a T cell-dependent autoimmune disease that arises from targeted destruction of the insulin-producing pancreatic beta cells. It is characterised by lifelong insulin dependence, an increased risk for the development of secondary complications, and reductions in the quality and duration of life.

Natural history and epidemiology The pathogenesis of type 1 diabetes begins early in life and progresses subclinically over a period of years [1]. During the course of the disease, failures in central and peripheral tolerance mechanisms allow for activation/expansion of autoreactive T cells [2], as well as other immune cell types, including B lymphocytes, as evidenced by the development of autoantibodies against islet autoantigens in the majority of patients [3]. While beta cells 
may persist in patients with established type 1 diabetes [4], clinical onset is thought to occur after loss or functional impairment of an estimated $50 \%$ or more of the beta cell mass [5]. Type 1 diabetes onset can occur at any time of life, even in old age [6]. However, $>50 \%$ of diagnoses are made in individuals under 20 years of age, at an incidence of $\sim 19$ per $100,000[7,8]$, making type 1 diabetes one of the most common childhood chronic diseases. Although paediatric-onset type 2 diabetes is becoming more common, type 1 diabetes continues to be the most common form in children, accounting for $\sim 90 \%$ of all paediatric diabetes diagnoses $[9,10]$.

Genetics Type 1 diabetes is a complex disease that arises through interactions between incompletely defined genetic, epigenetic and environmental factors, culminating in altered innate and adaptive immune functions. While it is a polygenic disease, the greatest genetic contribution is conferred by the HLA locus, specifically the $D R B 1 * 03-D Q B 1 * 0201$ (DR3) and $D R B 1 * 04-D Q B 1 * 0302$ (DR4) haplotypes. These HLA haplotypes are observed in $>90 \%$ of type 1 diabetic patients [11] and are associated with other autoimmune diseases [12], yet are carried by $\sim 40 \%$ of the Europid population. It is hypothesised that these high-risk alleles have been evolutionarily selected for their ability to present a broad range of microbial peptides to T cells [13]. However, this potential survival benefit has been seemingly retained at the cost of increased presentation of beta cell self-antigens. In contrast, certain HLA alleles, such as $D Q B 1^{*} 0503, * 0601, * 0602$ and *0603 are protective and are rarely observed in type 1 diabetic patients [14]. There are now $>40$ identified non-HLA loci that confer lesser levels of increased risk [15], and many of these mapped regions contain candidate genes that encode proteins governing immune responses (PTPN22, IL2RA, SH2B3, $P T P N 2, C T L A 4$ and $U B A S H 3 A$ ), including responses to beta cell antigens (INS) or to infectious agents (IFIHI) [3].

Environment In addition to genetic susceptibility, the pathogenesis of type 1 diabetes involves the contribution of environmental factors, which is reflected by disease concordances of $<100 \%$ among monozygotic twins [11]. Importantly, the global incidence of type 1 diabetes has been increasing by $2-3 \%$ per year, with the most rapid increase occurring among the youngest children $(<5$ years of age), an age group in which the incidence is anticipated to double within the next decade [16]. These epidemiological changes are too rapid to be accounted for by genetics alone and support the existence of new or changing environmental stressors that potentiate beta cell autoimmunity [17, 18]. Evidence that the proportion of 'high-risk' HLA genotypes in new-onset has decreased over time supports this idea [19, 20].

Viruses have long been considered an environmental trigger for type 1 diabetes in genetically susceptible individuals (reviewed in [21, 22]). While conclusive proof has remained elusive, epidemiological studies support a role for viruses, as the incidence of the type 1 diabetes varies with season and birth month $[23,24]$. Numerous studies have shown a higher rate of enterovirus infection among patients compared with controls (reviewed in [25]) and immunohistochemical staining studies have detected the enteroviral capsid protein VP1 at a higher frequency in islets of patients with new-onset diabetes compared with controls [26]. By serology, coxsackie B1 virus has been associated with an increased risk of beta cell autoimmunity [27] and in vitro human islet studies have reported that this virus preferentially infects human beta cells vs alpha cells, consistent with the selective beta cell demise seen in type 1 diabetes [28]. Finally, coxsackievirus has been shown to persist in the pancreas due to N-terminal deletions in its genome [29], consistent with a role in maintaining beta cell autoimmunity.

Dietary influences are also implicated in the pathogenesis of type 1 diabetes. These include the duration of breastfeeding as well as the timing of the introduction of cows' milk, cereal and gluten during childhood [30-33]. However, recent data from the BABYDIET and Trial to Reduce IDDM in the Genetically at Risk (TRIGR) studies indicate that diet alteration does not directly lower the incidence of seroconversion or type 1 diabetes $[34,35]$. The intestinal microbiome, governed by variables including host genetics, dietary influences and antibiotic use [36-38], has emerged as an important environmental influence. Several studies have measured differences in the microbiota of type 1 diabetic patients compared with healthy controls [39-42], as well as changes in the microbiota during the progression of diabetes, specifically between the time of seroconversion and clinical onset [43]. Beta cell autoimmunity has been associated with changes including reduced microbial diversity and a reduction in the bacterial phylum Firmicutes relative to Bacteroidetes $[42,44]$. These changes encompass a lower abundance of butyrate-producing genera, an important variable in the development, stability and function of regulatory T cells (Tregs), which are vital in maintaining immune tolerance (reviewed in [45]). Numerous studies have reported that diet and the composition of the gastrointestinal microbiome can increase intestinal permeability and promote a proinflammatory bias (reviewed in [46]). Notably, alterations in intestinal ultrastructure and intestinal barrier dysfunction have been associated with type 1 diabetes in humans (reviewed in [47]). Perhaps related to these observations, Oresic et al [48] identified metabolomic alterations among individuals who later progressed to type 1 diabetes.

It is possible that the rising incidence of type 1 diabetes and other autoimmune diseases may be associated with lifestyle changes that have occurred during the last century: a highfibre diet required for the maintenance of 'healthy' butyrateproducing microbiota is no longer the norm. As a consequence of the aforementioned non-HLA genetic susceptibility and/or increased or altered exposure to bacterial antigens, an elevated innate inflammatory state may now be present in a larger subset of the population, resulting in an overall larger 
pool of susceptible individuals, including individuals with sufficiently high baseline inflammation to bypass the historically requisite HLA haplotypes associated with the pathogenesis of diabetes.

While the best measures of disease activity are likely to be in the target tissue itself, human pancreatic islets are inaccessible in living people and are too diffuse to image. In contrast, peripheral blood is highly accessible for sequential sampling, making it a practical surrogate. Furthermore, between the blood, lymphatic system and site/s of active disease, there is a dynamic exchange of cells, proteins (cytokines/chemokines) and other factors (inflammatory lipids, microRNA [miRNA] and other molecules). As such, blood sampling offers a minimally invasive means to understand and follow human disease. Here, we aim to review studies of blood-based profiling in type 1 diabetes and place these within the context of the genetic and environmental factors implicated in the natural history of autoimmune diabetes.

\section{Markers of immune response to specific islet autoantigens}

Serum-based assays for autoantibodies to specific beta cell proteins, along with HLA genetics, have established the autoimmune pathogenesis of type 1 diabetes over the past 40 years [49]. Islet autoantibodies usually develop in a cluster, often early in life, and the presence of multiple islet autoantibodies confers a high risk of future diabetes, estimated at nearly $85 \%$ over a 15 -year span $[50,51]$. Despite detailed studies of various autoantibody targets, titres, isotypes and epitopes, it has proved difficult to predict the timing of clinical onset, highlighting the need for additional biomarkers.

Much effort has also been expended on measuring islet antigen-reactive immune cells in peripheral blood. Despite peptide-MHC tetramer technology, efforts to use information from islet-specific $\mathrm{CD}^{+} \mathrm{T}$-lymphocytes in peripheral blood have been hampered by their low precursor frequency. Similar approaches to measuring $\mathrm{CD} 8^{+} \mathrm{T}$ cells have made progress, especially those reactive against components of preproinsulin, although assays remain challenging because of the substantial blood volumes required and the practical need to use frozen/ thawed cells (reviewed in [52]). Similar to the situation with autoantibodies, the results from cellular assays highlight the need for additional biomarkers of pathophysiological mechanisms and clinical disease stage for type 1 diabetes.

\section{Blood-based signatures}

Direct gene expression profiling of whole blood Since the late 1990s there have been major technological developments in the fields of genomics and proteomics that now enable massively parallel analyses of RNA and protein. These advances have been coupled with significant developments in computational and bioinformatics, allowing meaningful interpretation of the complex datasets that these technologies generate. The use of these tools in cross-sectional and longitudinal analyses of blood samples collected from patients and controls has offered an unprecedented opportunity to gain new insights into human disease mechanisms and to identify new biomarkers of disease risk, progression and therapeutic response.

The most extensively applied blood-based approach strategy has been to directly measure the transcriptome of whole blood, peripheral blood mononuclear cells (PBMCs), or PBMC subpopulations of cases and controls. This direct transcriptomic strategy has made a significant clinical impact within the field of oncology, where it has been found sufficiently accurate to diagnose and classify acute lymphoblastic leukaemia subtypes, as well as to guide the selection of the most appropriate treatment modality (reviewed in [53]). When applied to infectious diseases, clinically useful and mechanistically informative transcriptional signatures have exhibited species- and strain-level specificity (reviewed in [54]). Different microbes generate pathogen-associated molecular patterns that interact with specific host pattern recognition receptors (PRRs, such as Toll-like-, NOD-like- and RIG-Ilike receptors) to activate unique combinations of signal transduction pathways, thus producing distinct transcriptional programs. Specific blood signatures have been measured in viral, bacterial and eukaryotic infectious diseases.

The transcriptional profiling of patient blood cells in autoimmune diseases has revealed important mechanistic insights that have advanced therapeutic interventions (reviewed in [55]). A role for type I IFN in human systemic lupus erythematosus was fostered by blood-based transcriptomics, as several studies observed the over-representation of IFN-regulated transcripts in patient PBMCs [55]. These and other observations, including elevated serum type I IFN levels and genetic association studies that identified susceptibility genes within the IFN pathway, have led to clinical trials of drugs targeting type I IFN in systemic lupus erythematosus [56]. In rheumatoid arthritis, transcriptional profiles of PBMCs can classify disease and predict responsiveness to infliximab treatment [55]. When applied to inflammatory bowel disease, bloodbased transcriptomics were sufficiently sensitive to distinguish Crohn's disease from ulcerative colitis and were able to identify individuals more likely to experience frequently relapsing disease [57, 58]. Furthermore, informative bloodbased transcriptional profiling studies have been reported for systemic-onset juvenile idiopathic arthritis and multiple sclerosis [55].

In type 1 diabetes, studies have described transcriptional profiles of whole blood [59-62], PBMCs [63-68] and immunocyte subsets [69-72]. Each sampling strategy has advantages and disadvantages (reviewed in [54]). However, the 
interpretation of blood-based transcriptional signatures should consider the cellular composition of the sample analysed and, as with other diseases, transcriptional signatures generated from these various sampling strategies have not been rigorously compared.

Kaizer et al [64] were among the first to compare gene expression profiles of paediatric type 1 diabetic patients with those of unrelated healthy controls. PBMCs of newly diagnosed patients exhibited an innate inflammatory transcriptional profile that included elevated IL1B, PTGS2, CXCL1, EGR2, EGR3 and TREM1 levels, which resolved in the months after diagnosis. Stechova et al [65] examined the transcriptional profiles of PBMCs isolated from paediatric diabetic patients, their healthy autoantibody-negative first-degree relatives and unrelated healthy controls. Interestingly, the greatest number of differentially expressed genes was identified when comparing the autoantibody-negative healthy relatives with the unrelated healthy controls, and the highest-scored immune response pathway was IL-1 signalling [65]. These findings are consistent with the results of a study that examined monozygotic twin pairs discordant for paediatric-onset type 1 diabetes [72]. Notably, the monocyte transcriptional profiles of twins discordant for diabetes were highly correlative with one another when compared with those of healthy monozygotic twin pairs and healthy singletons, and included increased expression of CCL2, CCL7 and CD9 [72]. Importantly, among the twins discordant for diabetes, the non-diabetic siblings studied had a low risk of disease progression based on the lack of autoantibodies [72]. The findings of this and other studies $[67,70]$ suggest that, as a consequence of common genetics and/or environmental factors, type 1 diabetic patients and their healthy relatives exhibit differential regulation of genes and pathways, particularly those consistent with increased activation of innate immune processes, in a manner distinct from that of unrelated healthy controls.

A study that investigated gene expression profiles of unfractionated whole blood in paediatric new-onset type 1 diabetic patients, their autoantibody-positive first-degree relatives, and unrelated healthy controls reported that, consistent with the hypothesis of a viral aetiology, an IFN-regulated signature (that included expression of IFI27, OASL, ISG15, IFIT3, GBP1, IFIT2, IFIT1, OAS3, STAT1, RSAD2, IFI44A) was found to be associated with patients and at-risk individuals [59]. Furthermore, blood-based transcriptomic analyses in two large longitudinal studies of at-risk infant cohorts, BABYDIET [68] and the Finnish Type 1 Diabetes Prediction and Prevention (DIPP) study [61], identified a transient IFN-driven signature in individuals prior to seroconversion and overt diabetes. More specifically, the IFN signature was found to be temporally preceded by recent respiratory infections, suggesting some degree of causality. In this context, it is possible that both those progressing to diabetes and their unaffected family members possess a heightened baseline innate inflammatory state and may tend towards a more vigorous response when virally challenged.

Several studies support that direct expression profiling of blood cells may be useful in stratifying disease progression both before and after the clinical onset of type 1 diabetes [62, 71]. Whole blood profiling studies of autoantibody-positive participants in the Diabetes Autoimmunity Study in the Young (DAISY) identified a set of transcripts implicated in lymphocyte activation and function $(B A C H 2, E I F 3 A, C D C 20$ and $T X N D C 5)$ whose expression levels were associated with various diabetes progression rates [62]. Among patients with new-onset diabetes, deviations from a healthy monocyte expression profile correlated with poorer glycaemic control, specifically, elevated $\mathrm{HbA}_{1 \mathrm{c}}$ and greater insulin requirements [71]. As such, stratification may prove valuable in identifying those at highest risk of diabetic complications.

\section{Serum/plasma-induced transcriptional analysis One} means by which immune cells mediate their actions is through the liberation of soluble mediators. The primary sites of immune activity in type 1 diabetes, namely the pancreatic islets and draining lymph nodes, represent a minimal fraction of body mass. Consequently, the concentration of cytokines, chemokines and other mediators may be high locally but too dilute in the periphery for direct measurement. Furthermore, measurement of a single or a few cytokines may be uninformative or even misleading because of important combinatorial effects. Our laboratory has employed an alternative approach to assay the extracellular milieu associated with diabetes susceptibility. This strategy, which was also used to study juvenile idiopathic arthritis and sepsis [73, 74], employs patient serum or plasma to induce transcriptional responses in a 'reporter' cell population. While various cell lines, neutrophils or islets [74-76] have been used in this type of assay, because of the breadth of expressed receptors, we have used cryopreserved PBMCs of a single well-controlled healthy blood donor as sensitive biosensors that transcriptionally respond to the dilute disease-associated factors in patient plasma. After co-culture, induced transcription is comprehensively measured with a genome-scale array and then the data are subjected to ontological analyses for quantitative interpretation in terms of inflammatory and regulatory immune activities [75, 77-81]. The identity of key serum mediators is then deduced from the nature of the regulated transcripts and this information is used to direct targeted follow-up studies. Since immunological responses are considered largely local events, participant immune cells may not be abundant in the periphery. This approach offers potential benefits over direct profiling of immune cells of patients and controls, including (1) restriction of the readout to that driven by the soluble compartment of potentially disease-associated factors, and (2) the responder PBMC population is derived from a single healthy donor, as opposed to immunocytes of type 1 diabetic patients, 
where heterogeneity in the measured transcriptional signatures, stemming from differing inherited combinations of genetic risk loci, could be expected. A possible limitation of the assay is the necessity to identify a representative responder cell population, as heterogeneity can be expected between fresh cells collected from multiple donors or successive draws of the same individual. Commercial providers now offer highly viable, cryopreserved PBMCs that are collected by aphaeresis. In a single draw of a healthy well-characterised donor, billions of cells, sufficient for thousands of assays, are collected. The availability of such cells greatly simplifies the process of testing a panel of candidates and identifying a representative PBMC donor.

In our first application of this approach [81], we observed that the plasma of autoantibody-positive individuals with new-onset type 1 diabetes induced a partially IL-1dependent signature consistent with immune activation and PRR ligand exposure relative to the plasma of unrelated healthy controls (uHC). This signature included induction of IL1B, CCL2, CCL7, ICAM1 and PTGS2. New-onset diabetic individuals lacking autoantibody titres were not different from their autoantibody-positive counterparts, indicating that the observed signature was independent of antibody status [81]. Similar to the post-onset resolution of the PBMC signature reported by Kaizer et al [64], signatures induced by the plasma of established diabetic patients $>10$ years post diagnosis were more similar to those of unrelated healthy controls lacking a family history of autoimmunity, suggesting that (1) our recentonset signature is related to active autoimmunity, and (2) established type 1 diabetes is immunologically quiescent relative to the immediate post-onset period. In our study we longitudinally analysed pre-onset samples from individuals who progressed to diabetes, and found that the signature associated with recent-onset type 1 diabetes preceded both the development of autoantibodies and clinical diagnosis by as much as 5 years [81]. Consistent with these observations, Jackson et al observed that type 1 diabetes plasma also induces a proinflammatory, IL-1 biased signature when using cultured human islets as the responder population [76].

Given their common IL-1 bias, we compared the reported 46 transcript signature induced by the plasma of patients with juvenile idiopathic arthritis [73] to that induced by type 1 diabetes plasma samples. While sharing some identity, the signatures were found to be distinct [81]. We have also applied this approach to H1N1 influenza, bacterial pneumonia, and cystic fibrosis with airway Pseudomonas aeruginosa colonisation [80], and have identified specific, mechanistically informative signatures for each disease state.

Our group has also used plasma-induced transcription to investigate immune states within type 1 diabetes families (i.e. siblings of type 1 diabetes probands) and how these temporally change with either progression or non-progression to diabetes. Siblings of patients with type 1 diabetes have an estimated $6 \%$ probability of developing diabetes and the majority of those who progress will possess a high-risk DR3 and/ or DR4 HLA haplotype [11, 82]. Therefore, we crosssectionally examined patients with recent-onset diabetes, their healthy autoantibody-negative siblings who either possessed or lacked DR3 and/or DR4 HLA haplotypes (termed high-risk siblings [HRS] or low-risk siblings [LRS], respectively), as well as uHC [78]. While the plasma of patients with recentonset diabetes induced a distinct signature relative to that of the LRS, HRS and uHC, the signatures of these healthy cohorts were also distinct from one another. Expectedly, the recent-onset diabetes and HRS cohorts possessed the most similar signatures, in accordance with their overlapping HLA-determined susceptibility. We predicted that given the low genetic risk of the LRS, this cohort would be most similar to the uHC, but, surprisingly, the signatures of these two cohorts were most distinct. Notably, the LRS signature exhibited the most robust induction of innate inflammatory transcripts (e.g. IL1B, CCL2, CCL3, CCL7, CXCL1, CXCL2, CXCL3, $C D 14$ and TREM1). Pathway analyses revealed a continuum of immune states across the four cohorts. Relative to the uHC, an elevated, partially IL-1 dependent inflammatory state was present in patients with recent-onset type 1 diabetes and their unaffected siblings. This familial inflammatory state displayed greater evidence of being immunoregulated among the HRS compared with the LRS, as the HRS signature exhibited more robust induction of IL-10/TGF- $\beta$-dependent transcripts (e.g. TGFBR2, SMAD9, SMAD5, SKI, SKIL SMURF1, SMURF2, FCGR2B, PIAS1, CASP8, and LGALS3). This observation suggests that among HRS, where possession of high-risk HLA haplotypes increases the likelihood of an adaptive response and diabetes progression, immunoregulatory mechanisms are more active. The plasma of patients with recentonset type 1 diabetes (ROT1D) induced signatures intermediate between those of LRS and HRS, such that inflammation decreased and regulation increased across the LRS $\rightarrow$ $\mathrm{ROT1D} \rightarrow \mathrm{HRS} \rightarrow \mathrm{uHC}$ continuum.

The dependencies of this continuum on IL-1, IL-10 and TGF- $\beta$ were validated through in vitro studies whereby cocultures were spiked with either cytokines or receptorblocking antibodies [78]. Multiplex cytokine analyses of LRS, HRS and patients with recent-onset diabetes failed to detect differences among these three type 1 diabetes family cohorts; however, significantly elevated levels of IL- $1 \alpha$, IL12p40, CCL2, CCL3 and CCL4 were detected in the diabetic patients and their healthy autoantibody-negative siblings relative to the uHC [78]. While plasma-induced signature analysis was able to differentiate the four cohorts, the selected 25-plex ELISA was not, highlighting the ability of the bioassay to capture the combinatorial effect of the plasma-borne factors.

Consistent with several of the aforementioned direct bloodbased transcriptomic studies [64, 65, 72], plasma-induced transcriptional analyses support the existence of a partially IL-1- 
dependent innate inflammatory state in type 1 diabetes families that is consistent with PRR ligand exposure (in particular, bacterial endotoxin exposure) and nuclear factor- $\mathrm{B}$ activation, but independent of HLA haplotype, autoantibody status and diabetes progression. This is further supported by other studies using more traditional approaches. Although results have been inconsistent as regards peripheral cytokine and chemokine levels in type 1 diabetes - perhaps because of the common practice of using healthy siblings as controls instead of unrelated individuals - elevated peripheral levels of IL-1 have been reported in diabetic individuals [83-85]. Furthermore, compared with controls, leucocytes from diabetic patients and their family members exhibit elevated responsiveness, as evidenced by the liberation of higher levels of IL-1 upon mitogen stimulation in vitro [84-87]. Both type 1 diabetic patients and their family members have quantitatively fewer peripheral dendritic cells than healthy controls, but produce more IFN $\alpha$ in response to Toll-like receptor-9 stimulation than controls [88]. Furthermore, global serum-based proteomic analysis of type 1 diabetic patients and unrelated healthy controls has provided corroborating evidence of an innate inflammatory state associated with autoimmune diabetes [89].

\section{Temporal induction of immunoregulation in non-} progressors We have also used plasma-induced transcription to temporally study the extracellular milieu of healthy siblings of diabetic patients during progression or non-progression to type 1 diabetes [78]. These studies were facilitated by the development of a gene ontology-based scoring algorithm to quantitatively capture overall changes in immune balance. Since the regulated transcripts identified in the cross-sectional studies could be broadly annotated as being 'inflammatory' or 'regulatory', we quantitatively scored signatures with a composite inflammatory index (I.I.com) determined by calculating an average ratio of the mean log intensity of the inflammatory genes vs the mean log intensity of the regulatory genes [78]. Among those HRS and LRS progressing to type 1 diabetes, we consistently observed temporal increases in inflammatory activity with coincident reductions in regulatory activity, resulting in overall increases in I.I.com [78] (Fig. 1a, d, g, j). However, this crescendo was not linear, but, rather, was observed to have fluctuations, possibly representing the flares and remissions that have been postulated in type 1 diabetes [90]. Perhaps expectedly, an increase in inflammatory bias was observed in association with, or prior to, the detection of autoantibodies. Notably, the opposite was also observed, with increases in regulatory bias being associated with decreases in the number of detected autoantibodies (Fig. 1b, e, h, k). Longitudinal analysis of persistently autoantibody-negative HRS revealed temporal decreases in the induction of transcripts annotated as inflammatory, and increases in those annotated as regulatory (Fig. 1c, $\mathrm{f}, \mathrm{i}, \mathrm{l})$. Accordingly, this was reflected by overall decreases in I.I.com. These data indicate that HRS who do not progress to type 1 diabetes temporally regulate the familial inflammatory state, suggesting the possibility of age-dependent alterations in the functional status of regulatory T cells (Tregs). Indeed, we have shown a significant correlation between decreased inflammatory bias and an increased percentage of activated Tregs over time in HRS non-progressors. This inverse correlation was not significantly observed in LRS. Taken together, these data suggest that the innate inflammatory state present in type 1 diabetes families is more actively regulated in an agedependent manner and in the presence of high-risk HLA haplotypes, offering mechanistic insight as to why human autoimmune diabetes susceptibility declines with age [78].

Other studies have also provided evidence of active homeostatic mechanisms that counter-regulate the inherited risk of progressing to type 1 diabetes. Some diabetes family members never progress to clinical onset of type 1 diabetes, despite the development of autoantibodies. Furthermore, in firstdegree relatives of diabetic patients lacking autoantibodies the balance of inflammatory and regulatory T cells is intermediate to that in patients with recent-onset type 1 diabetes and unrelated healthy controls [91]. The capacity of a given individual to manage the familial innate inflammatory state and successfully maintain tolerance to islets is likely to stem from inheritance of variable combinations of genetic predisposition in concert with a lifetime course of environmental events, including viral infections, diet and the gastrointestinal microbiome [17, 18, 92].

\section{Other blood-based signatures in type 1 diabetes: proteomics, miRNA, circulating beta cell DNA}

Serum-based proteomics also promises new insights into the pathogenesis of type 1 diabetes [93], but its use is limited by the wide range of physiological levels of proteins, the multiple functions of a given protein in common cellular pathways and the significant proteolytic processing and post-translational modifications of protein precursors. Despite these limitations, the sera from type 1 diabetic patients showed higher expression of inflammatory mediators such as C-reactive protein and serum amyloid $\mathrm{A}$, adiponectin and insulin-like growth factor binding protein 2 , but lower expression of TGF- $\beta$ and myeloperoxidase compared with the sera from healthy controls [93]. Using liquid chromatography-mass spectrometry and a validation strategy that used proteolytic peptides as surrogates for candidate proteins, Zhang et al confirmed that the expression of 24 proteins involved in innate inflammation and complement cascade activation is altered in sera from type 1 diabetic patients compared with controls [89]. More recently, longitudinal serum proteomic profiles have been measured in children with a genetic risk of developing diabetes to establish changes associated with seroconversion and progression to clinical onset [94]. In this study, children progressing to onset 

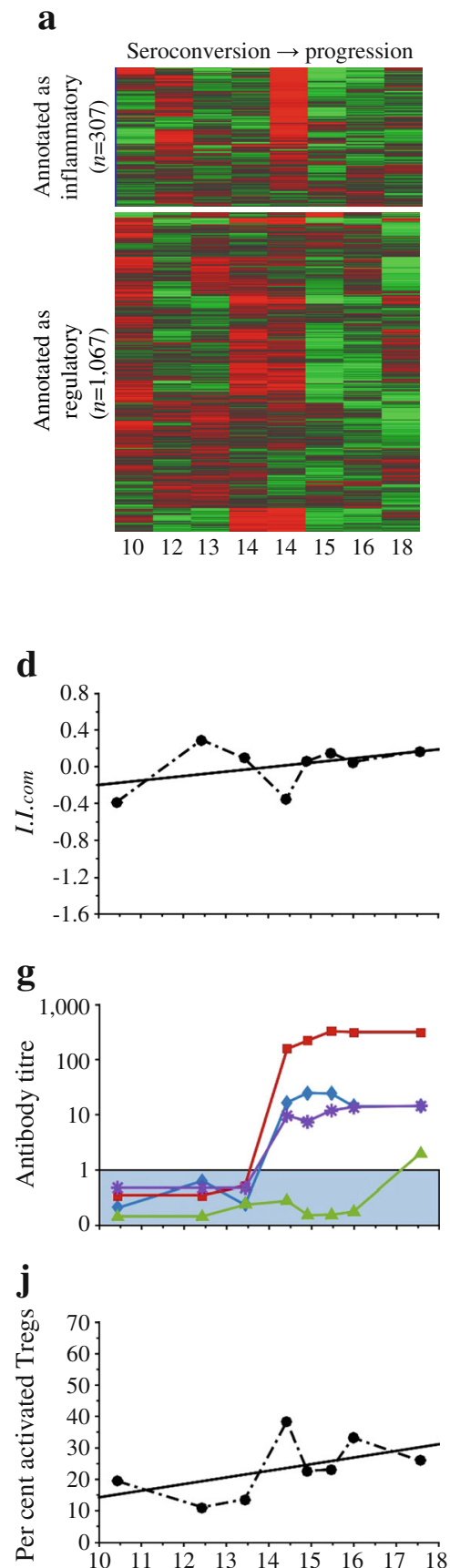

b

Seroconversion $\rightarrow$ no progression
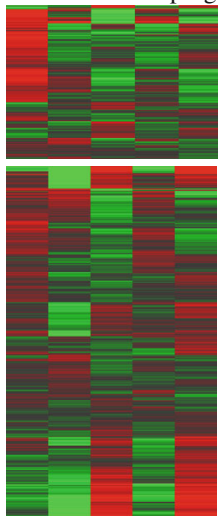

$\begin{array}{lllll}12 & 13 & 14 & 15 & 16\end{array}$

Age (years) c

No seroconversion $\rightarrow$ no progression
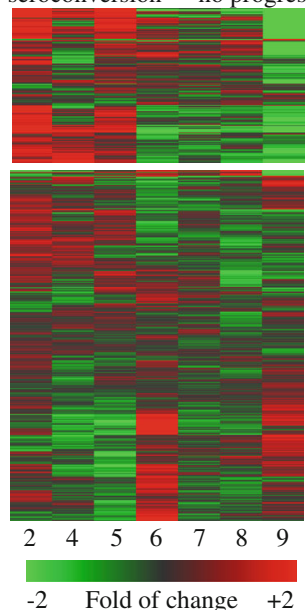

f

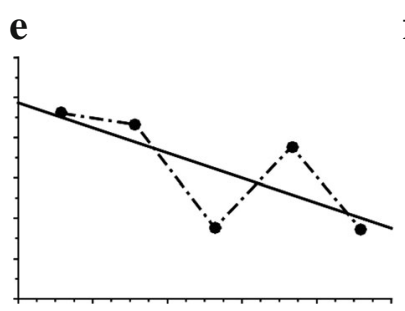

h $\quad$ i

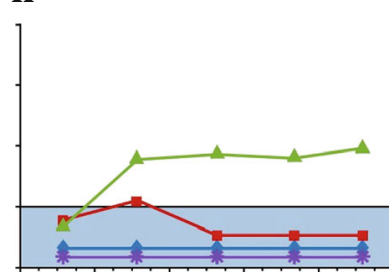

$\mathbf{k}$

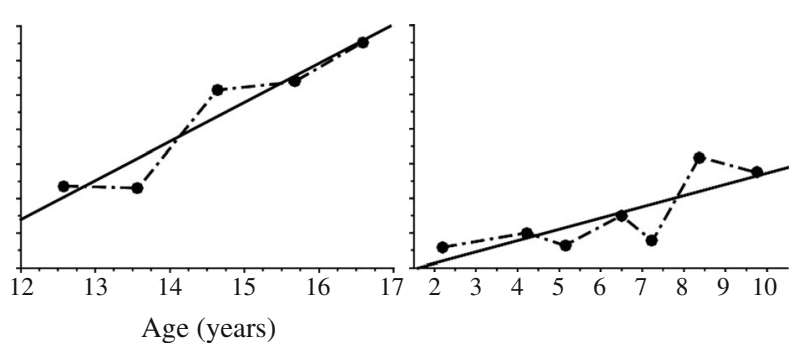

Fig. 1 Longitudinal analyses of diabetes progression or non-progression among HRS of type 1 diabetes probands with integration of multiple biomarkers. (a, d, g, j) Analysis of a type 1 diabetes progressor. (b, e, $\mathbf{h}, \mathbf{k})$ Analysis of an autoantibody-positive non-progressor. (c, f, i, l) Analysis of an autoantibody-negative non-progressor. (a-c) Heat maps illustrating plasma-induced transcription. Expression levels of the 307 transcripts annotated as being inflammatory and 1,067 transcripts annotated as being regulatory genes that were identified in [78] and used for temporal scoring of signatures. (d-f) Temporal plot of the composite inflammatory index (I.I.com) derived from the ontology-based scoring of the plasma-induced signature analysis described in [78]. This measure reflects the average induction of inflammatory transcripts vs the average induction of regulatory transcripts. (g-i) Temporal analysis of autoantibody titres (as described in [109]), plotted on a $\log _{10}$ scale as fold change relative to the upper limit of normal controls. Blue shaded area $<1=$ antibody-negative. Blue line, GAD; red line, IA2; green line, IAA; purple line, ZnT8. (j-l) Longitudinal analyses of the percentage of activated Tregs among the total Tregs in the periphery. As described in [110], resting and activated $\mathrm{CD}^{+}$Tregs were respectively defined as $\mathrm{CD}_{5} 5 \mathrm{RA}^{+} /$FoxP $^{\text {low }}$ and $\mathrm{CD} 45 \mathrm{RA}^{-} /$FoxP $3{ }^{\text {high }}$. The relationship between percentage activated Tregs and age was not significant in the type 1 diabetes progressor illustrated in (j) (slope $=0.02, p=0.19, R^{2}=0.26$ ); but was found significant in the non-progressors illustrated in $(\mathbf{k})$ (slope $\left.=0.11, p=0.01, R^{2}=0.90\right)$ and (l) $\left(\right.$ slope $\left.=0.03, p=0.04, R^{2}=0.62\right)$ 
had distinct proteomic profiles, perhaps offering an adjunctive means of predicting type 1 diabetes beyond autoantibody titres. Direct proteomic analysis of endocrine and exocrine pancreas tissues has identified protein panels that are uniquely regulated in autoantibody-positive individuals and type 1 diabetic patients [95]. Furthermore, these analyses were able to differentiate between type 1 diabetes and type 2 diabetes, providing a foundation for studies aimed at evaluating pancreasspecific proteins in peripheral blood samples that may serve as biomarkers for the early detection of type 1 diabetes [95].

Circulating miRNAs, which are small, noncoding RNA molecules that negatively regulate protein expression at the post-translational level, have also been studied as potential biomarkers in autoimmune diseases (reviewed in [96]). Distinct circulating miRNA profiles have been measured in children recently diagnosed with type 1 diabetes vs controls and identified miRNA linked to apoptosis and beta cell function [97]. In addition, the miRNA expression profiles of PBMCs of type 1 diabetic individuals have been reported to be distinct from those of PBMCs of healthy controls [98]. The application of miRNA profiling to those at risk of developing type 1 diabetes has yet to be reported.

Methylation is a means of silencing genes, and beta cellderived demethylated insulin (INS) DNA in serum or plasma has been investigated as a valuable biomarker of beta cell death. Elevated circulating levels of beta cell-derived unmethylated INS DNA (relative to methylated INS DNA) have been shown to be reflective of beta cell death and newonset type 1 diabetic patients exhibit higher circulating unmethylated INS DNA than healthy controls [99]. This approach has since been technically refined and applied to additional patient and control cohorts [100,101], including studies of the pre-onset period, during which elevated levels of unmethylated INS DNA were detected. In a clinical trial setting, decreases in unmethylated INS DNA levels were associated with improved beta cell function as measured by stimulated C-peptide in patients with new-onset diabetes who were receiving anti-CD3 monoclonal antibody [102]. These findings suggest that measurement of unmethylated INS DNA may have utility in monitoring the efficacy of therapeutic interventions.

\section{Reconciliation of blood signature studies with the natural} history of type 1 diabetes The findings of studies that used these blood-based approaches both support and augment our working models of the pathogenesis of diabetes (Fig. 2). Many studies have now identified an elevated inflammatory state associated with type 1 diabetes $[64,65,67,70,72,78$,

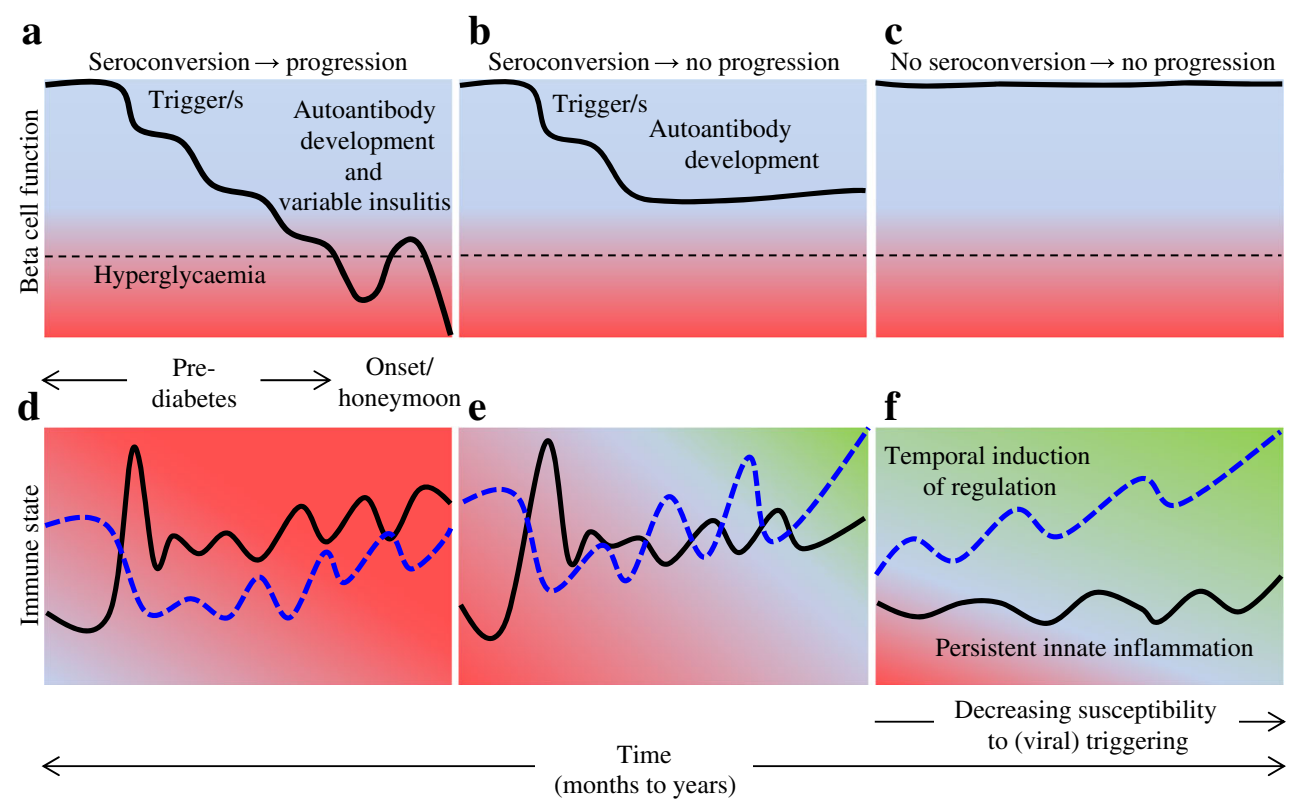

Fig. 2 Models of type 1 diabetes progression or non-progression. (a, d) Disease progression of an HRS, equivalent to the individual illustrated in Fig. 1a, d, g, j. (a-c) A nonlinear decay in beta cell mass following environmental triggering of autoimmunity in a genetically susceptible individual (derived from the model originally proposed by Eisenbarth [111] and subsequently modified $[90,112])$. (d-f) The immune state in terms of proinflammatory activity (black line) or immune-regulatory activity (blue line) reflected by the plasma-induced signatures illustrated in Fig. 1. Shading reflects overall bias captured by I.I.com (green, regulatory; red, inflammatory). (b, e) Disease non-progression in an antibody-positive high HRS of a type 1 diabetes proband, equivalent to the individual illustrated in Fig. 1b, e, h, k. This individual experienced disruption followed by restoration of the balance between inflammatory and regulatory processes. (c, f) Disease non-progression in an antibody-negative HRS of a type 1 diabetes proband, equivalent to the individual illustrated in Fig. 1 c, f, i, l. Our studies support the existence of an endogenous elevated innate inflammatory state in type 1 diabetes families that is counter-regulated in an age-dependent manner resulting in a state of regulated susceptibility. Prior to the generation of a robust immunoregulatory compartment (reflected by green shading in lower panel), there is a window of susceptibility of (viral) disease triggering, which temporally diminishes 
$81,84,87]$ that is consistent with a genetically controlled hypersensitivity towards and/or an increased exposure to PRR ligands potentially mediated by microbiome alterations and intestinal hyper-permeability. This may indeed be the susceptible state as proposed by the 'fertile field hypothesis', whereby interactions with microbes induce a condition primed for the triggering of autoimmunity [103]. Among individuals at risk of developing type 1 diabetes, studies have identified age-dependent endogenous regulatory mechanisms that counter-regulate the familial innate state, reflected in part by our observations of temporal increases in the percentage of activated Tregs in the HRS who do not progress to clinical onset of diabetes [78]. Viral infections may further elevate the inflammatory state, especially early in life before the establishment of a robust regulatory compartment, allowing for the activation and expansion of autoreactive T cells [103]. Relevant to the reports of transient IFN signatures [61, 68] and the temporal fluctuations observed in our longitudinal plasma-induced signature analyses [78] (Fig. 1), exposure of Tregs to inflammatory inputs can impair their suppressive capacity $[104,105]$. Thus, in addition to genetic variation and the endogenous innate state, additional inflammatory signals, such as those mediated by viral infections, especially in younger individuals, may impair compensatory regulatory mechanisms and promote autoimmunity. This scenario is modelled by the BioBreeding (BB) DR rat, which, similar to siblings of type 1 diabetes probands, exhibit an underlying innate inflammatory state with temporally induced immunoregulatory processes $[79,106]$. BBDR rats are not spontaneously diabetic, but autoimmune diabetes can be triggered in young animals by immunological perturbations that include viral infection (reviewed in [77]). Our studies show that the susceptibility of BBDR rats to virally triggered diabetes declines with age and is coincident with the temporal acquisition of an IL-10- and TGF- $\beta$-mediated immunoregulated state [77, $79,106]$. In humans, prevention of type 1 diabetes will be fostered by the accurate prediction of those who will progress to onset and the development of therapies that can reinforce the default protective regulatory response.

Autoantibodies were first described in type 1 diabetes over 40 years ago [107], and after decades of refinement they are the best biomarker of future clinical onset. However, they have limitations in revealing the timing of disease onset. Compared with autoantibody measurements, the application of systems-based approaches to type 1 diabetes is in its infancy, yet it holds the promise of identifying new biomarkers to augment autoantibodies. Blood-based signatures may be useful not only in predicting and staging the progression of diabetes, but also for monitoring changes in the islet-specific immune state during therapeutic interventions [108] and serving as surrogate outcomes to shorten the duration of clinical trials. Going forward, it is to be expected that these systemsbased phenotypic measures will be tied to genetic and epigenetic data, fostering an even deeper understanding of processes that govern the pathogenesis of type 1 diabetes.

Acknowledgements The authors thank Rhonda Geoffrey, B.S., Shuang Jia, M.S., Mary Kaldunski, B.S., and Joanna Kramer, B.S. within the Department of Pediatrics, The Medical College of Wisconsin, for data preparation.

Funding The work was supported by The Juvenile Diabetes Research Foundation International (grants 5-2012-220, 17-2012-621, 2-SRA2015-109-Q-R to MJH); American Diabetes Association (grant 7-12BS-075 to MJH); National Institutes of Health (grants R01AI078713, DP3DK098161 to MJH and R00DK077443, DP3DK097605, R21AI110963 to YC); The Children's Research Institute of Children's Hospital of Wisconsin (grants FP6477 to SMC and FP7674 to MJH); and The Children's Hospital of Wisconsin Foundation.

Duality of interest All authors have read and understand the policy on disclosure of conflicts of interest. The authors have no conflicts of interests to declare.

Contribution statement $\mathrm{SMC}, \mathrm{YC}, \mathrm{WAH}$ and $\mathrm{MJH}$ researched, wrote and revised the manuscript. All authors approved the version to be published.

\section{References}

1. Kimpimaki T, Kupila A, Hamalainen AM et al (2001) The first signs of beta-cell autoimmunity appear in infancy in genetically susceptible children from the general population: the Finnish Type 1 Diabetes Prediction and Prevention Study. J Clin Endocrinol Metab 86:4782-4788

2. Todd JA (2010) Etiology of type 1 diabetes. Immunity 32:457467

3. Morran MP, Vonberg A, Khadra A, Pietropaolo M (2015) Immunogenetics of type 1 diabetes mellitus. Mol Asp Med 42: $42-60$

4. Meier JJ, Bhushan A, Butler AE, Rizza RA, Butler PC (2005) Sustained beta cell apoptosis in patients with long-standing type 1 diabetes: indirect evidence for islet regeneration? Diabetologia 48:2221-2228

5. Akirav E, Kushner JA, Herold KC (2008) Beta-cell mass and type 1 diabetes: going, going, gone? Diabetes 57:2883-2888

6. Thunander M, Petersson C, Jonzon K et al (2008) Incidence of type 1 and type 2 diabetes in adults and children in Kronoberg, Sweden. Diabetes Res Clin Pract 82:247-255

7. Haller MJ, Atkinson MA, Schatz D (2005) Type 1 diabetes mellitus: etiology, presentation, and management. Pediatr Clin N Am 52:1553-1578

8. Maahs DM, West NA, Lawrence JM, Mayer-Davis EJ (2010) Epidemiology of type 1 diabetes. Endocrinol Metab Clin N Am 39:481-497

9. Liese AD, D'Agostino RB Jr, Hamman RF et al (2006) The burden of diabetes mellitus among US youth: prevalence estimates from the SEARCH for Diabetes in Youth Study. Pediatrics 118: $1510-1518$

10. Dabelea D, Bell RA, D'Agostino RB Jr et al (2007) Incidence of diabetes in youth in the United States. JAMA 297:2716-2724

11. Steck AK, Rewers MJ (2011) Genetics of type 1 diabetes. Clin Chem 57:176-185 
12. Gough SC, Simmonds MJ (2007) The HLA region and autoimmune disease: associations and mechanisms of action. Curr Genomics 8:453-465

13. Mangalam AK, Taneja V, David CS (2013) HLA class II molecules influence susceptibility versus protection in inflammatory diseases by determining the cytokine profile. J Immunol 190: 513-518

14. Redondo MJ, Fain PR, Eisenbarth GS (2001) Genetics of type 1A diabetes. Recent Prog Horm Res 56:69-89

15. Onengut-Gumuscu S, Chen WM, Burren O et al (2015) Fine mapping of type 1 diabetes susceptibility loci and evidence for colocalization of causal variants with lymphoid gene enhancers. Nat Genet 47:381-386

16. Patterson CC, Gyurus E, Rosenbauer J et al (2012) Trends in childhood type 1 diabetes incidence in Europe during 19892008: evidence of non-uniformity over time in rates of increase. Diabetologia 55:2142-2147

17. Vaarala O, Atkinson MA, Neu J (2008) The "perfect storm" for type 1 diabetes: the complex interplay between intestinal microbiota, gut permeability, and mucosal immunity. Diabetes 57:25552562

18. Atkinson MA, Chervonsky A (2012) Does the gut microbiota have a role in type 1 diabetes? Early evidence from humans and animal models of the disease. Diabetologia 55:2868-2877

19. Fourlanos S, Varney MD, Tait BD et al (2008) The rising incidence of type 1 diabetes is accounted for by cases with lower-risk human leukocyte antigen genotypes. Diabetes Care 31:15461549

20. Borchers AT, Uibo R, Gershwin ME (2010) The geoepidemiology of type 1 diabetes. Autoimmun Rev 9:A355-A365

21. Schneider DA, von Herrath MG (2013) Viruses and type 1 diabetes: a dynamic labile equilibrium. Diabetes Manag (Lond) 3:217223

22. Jaidane H, Sauter P, Sane F, Goffard A, Gharbi J, Hober D (2010) Enteroviruses and type 1 diabetes: towards a better understanding of the relationship. Rev Med Virol 20:265-280

23. Kahn HS, Morgan TM, Case LD et al (2009) Association of type 1 diabetes with month of birth among U.S. youth: the SEARCH for Diabetes in Youth Study. Diabetes Care 32:2010-2015

24. Moltchanova EV, Schreier N, Lammi N, Karvonen M (2009) Seasonal variation of diagnosis of type 1 diabetes mellitus in children worldwide. Diabet Med 26:673-678

25. Yeung WC, Rawlinson WD, Craig ME (2011) Enterovirus infection and type 1 diabetes mellitus: systematic review and metaanalysis of observational molecular studies. BMJ 342:d35

26. Richardson SJ, Leete P, Bone AJ, Foulis AK, Morgan NG (2013) Expression of the enteroviral capsid protein VP1 in the islet cells of patients with type 1 diabetes is associated with induction of protein kinase R and downregulation of Mcl-1. Diabetologia 56: 185-193

27. Laitinen $\mathrm{OH}$, Honkanen H, Pakkanen $\mathrm{O}$ et al (2014) Coxsackievirus B1 is associated with induction of beta-cell autoimmunity that portends type 1 diabetes. Diabetes 63:446-455

28. Anagandula M, Richardson SJ, Oberste MS et al (2014) Infection of human islets of Langerhans with two strains of Coxsackie B virus serotype 1: assessment of virus replication, degree of cell death and induction of genes involved in the innate immunity pathway. J Med Virol 86:1402-1411

29. Tracy S, Smithee S, Alhazmi A, Chapman N (2015) Coxsackievirus can persist in murine pancreas by deletion of $5^{\prime}$ terminal genomic sequences. J Med Virol 87:240-247

30. Gerstein HC (1994) Cow's milk exposure and type I diabetes mellitus. A critical overview of the clinical literature. Diabetes Care 17:13-19
31. Knip M, Virtanen SM, Seppa K et al (2010) Dietary intervention in infancy and later signs of beta-cell autoimmunity. N Engl J Med 363:1900-1908

32. Norris JM, Barriga K, Klingensmith G et al (2003) Timing of initial cereal exposure in infancy and risk of islet autoimmunity. JAMA 290:1713-1720

33. Ziegler AG, Schmid S, Huber D, Hummel M, Bonifacio E (2003) Early infant feeding and risk of developing type 1 diabetesassociated autoantibodies. JAMA 290:1721-1728

34. Beyerlein A, Chmiel R, Hummel S, Winkler C, Bonifacio E, Ziegler AG (2014) Timing of gluten introduction and islet autoimmunity in young children: updated results from the BABYDIET study. Diabetes Care 37:e194-e195

35. Knip M, Akerblom HK, Becker D et al (2014) Hydrolyzed infant formula and early beta-cell autoimmunity: a randomized clinical trial. JAMA 311:2279-2287

36. Benson AK, Kelly SA, Legge R et al (2010) Individuality in gut microbiota composition is a complex polygenic trait shaped by multiple environmental and host genetic factors. Proc Natl Acad Sci U S A 107:18933-18938

37. Turnbaugh PJ, Hamady M, Yatsunenko T et al (2009) A core gut microbiome in obese and lean twins. Nature 457:480-484

38. Khachatryan ZA, Ktsoyan ZA, Manukyan GP, Kelly D, Ghazaryan KA, Aminov RI (2008) Predominant role of host genetics in controlling the composition of gut microbiota. PLoS One 3, e3064

39. Murri M, Leiva I, Gomez-Zumaquero JM et al (2013) Gut microbiota in children with type 1 diabetes differs from that in healthy children: a case-control study. BMC Med 11:46

40. Mejia-Leon ME, Petrosino JF, Ajami NJ, Dominguez-Bello MG, de la Barca AM (2014) Fecal microbiota imbalance in Mexican children with type 1 diabetes. Sci Rep 4:3814

41. Endesfelder D, zu Castell W, Ardissone A et al (2014) Compromised gut microbiota networks in children with antiislet cell autoimmunity. Diabetes 63:2006-2014

42. Giongo A, Gano KA, Crabb DB et al (2011) Toward defining the autoimmune microbiome for type 1 diabetes. ISME J 5:82-91

43. Kostic AD, Gevers D, Siljander H et al (2015) The dynamics of the human infant gut microbiome in development and in progression toward type 1 diabetes. Cell Host Microbe 17:260-273

44. Brown CT, Davis-Richardson AG, Giongo A et al (2011) Gut microbiome metagenomics analysis suggests a functional model for the development of autoimmunity for type 1 diabetes. PLoS One 6, e25792

45. Hoeppli RE, Wu D, Cook L, Levings MK (2015) The environment of regulatory $\mathrm{T}$ cell biology: cytokines, metabolites, and the microbiome. Front Immunol 6:61

46. Gomes AC, Bueno AA, de Souza RG, Mota JF (2014) Gut microbiota, probiotics and diabetes. Nutr J 13:60

47. Li X, Atkinson MA (2015) The role for gut permeability in the pathogenesis of type 1 diabetes - a solid or leaky concept? Pediatr Diabetes 16:485-492

48. Oresic M, Simell S, Sysi-Aho M et al (2008) Dysregulation of lipid and amino acid metabolism precedes islet autoimmunity in children who later progress to type 1 diabetes. J Exp Med 205: 2975-2984

49. Winter WE, Schatz DA (2011) Autoimmune markers in diabetes. Clin Chem 57:168-175

50. Kupila A, Keskinen P, Simell T et al (2002) Genetic risk determines the emergence of diabetes-associated autoantibodies in young children. Diabetes 51:646-651

51. Ziegler AG, Rewers M, Simell O et al (2013) Seroconversion to multiple islet autoantibodies and risk of progression to diabetes in children. JAMA 309:2473-2479

52. Roep BO, Peakman M (2012) Antigen targets of type 1 diabetes autoimmunity. Cold Spring Harb Perspect Med 2:a007781 
53. Roberts KG, Mullighan CG (2015) Genomics in acute lymphoblastic leukaemia: insights and treatment implications. Nat Rev Clin Oncol 12:344-357

54. Blankley S, Berry MP, Graham CM, Bloom CI, Lipman M, O'Garra A (2014) The application of transcriptional blood signatures to enhance our understanding of the host response to infection: the example of tuberculosis. Philos Trans R Soc Lond B Biol Sci 369:20130427

55. Pascual V, Chaussabel D, Banchereau J (2010) A genomic approach to human autoimmune diseases. Annu Rev Immunol 28: 535-571

56. Kirou KA, Gkrouzman E (2013) Anti-interferon alpha treatment in SLE. Clin Immunol 148:303-312

57. Burczynski ME, Peterson RL, Twine NC et al (2006) Molecular classification of Crohn's disease and ulcerative colitis patients using transcriptional profiles in peripheral blood mononuclear cells. J Mol Diagn 8:51-61

58. Lee JC, Lyons PA, McKinney EF et al (2011) Gene expression profiling of CD8 $+\mathrm{T}$ cells predicts prognosis in patients with Crohn disease and ulcerative colitis. J Clin Invest 121:4170-4179

59. Reynier F, Pachot A, Paye M et al (2010) Specific gene expression signature associated with development of autoimmune type-I diabetes using whole-blood microarray analysis. Genes Immun 11: 269-278

60. Elo LL, Mykkanen J, Nikula T et al (2010) Early suppression of immune response pathways characterizes children with prediabetes in genome-wide gene expression profiling. J Autoimmun 35: $70-76$

61. Kallionpaa H, Elo LL, Laajala E et al (2014) Innate immune activity is detected prior to seroconversion in children with HLAconferred type 1 diabetes susceptibility. Diabetes 63:2402-2414

62. Jin Y, Sharma A, Bai S et al (2014) Risk of type 1 diabetes progression in islet autoantibody-positive children can be further stratified using expression patterns of multiple genes implicated in peripheral blood lymphocyte activation and function. Diabetes 63:2506-2515

63. Rassi DM, Junta CM, Fachin AL et al (2006) Metabolism genes are among the differentially expressed ones observed in lymphomononuclear cells of recently diagnosed type 1 diabetes mellitus patients. Ann N Y Acad Sci 1079:171-176

64. Kaizer EC, Glaser CL, Chaussabel D, Banchereau J, Pascual V, White PC (2007) Gene expression in peripheral blood mononuclear cells from children with diabetes. J Clin Endocrinol Metab 92:3705-3711

65. Stechova K, Kolar M, Blatny R et al (2012) Healthy first-degree relatives of patients with type 1 diabetes exhibit significant differences in basal gene expression pattern of immunocompetent cells compared to controls: expression pattern as predeterminant of autoimmune diabetes. Scand J Immunol 75:210-219

66. Evangelista AF, Collares CV, Xavier DJ et al (2014) Integrative analysis of the transcriptome profiles observed in type 1 , type 2 and gestational diabetes mellitus reveals the role of inflammation. BMC Med Genomics 7:28

67. Jin Y, Sharma A, Carey C et al (2013) The expression of inflammatory genes is upregulated in peripheral blood of patients with type 1 diabetes. Diabetes Care 36:2794-2802

68. Ferreira RC, Guo H, Coulson RM et al (2014) A type I interferon transcriptional signature precedes autoimmunity in children genetically at risk for type 1 diabetes. Diabetes 63:2538-2550

69. Orban T, Kis J, Szereday L et al (2007) Reduced CD4+ T-cellspecific gene expression in human type 1 diabetes mellitus. $\mathrm{J}$ Autoimmun 28:177-187

70. Padmos RC, Schloot NC, Beyan H et al (2008) Distinct monocyte gene-expression profiles in autoimmune diabetes. Diabetes 57 : $2768-2773$
71. Irvine KM, Gallego P, An X et al (2012) Peripheral blood monocyte gene expression profile clinically stratifies patients with recent-onset type 1 diabetes. Diabetes 61:1281-1290

72. Beyan H, Drexhage RC, van der Heul Nieuwenhuijsen L et al (2010) Monocyte gene-expression profiles associated with childhood-onset type 1 diabetes and disease risk: a study of identical twins. Diabetes 59:1751-1755

73. Pascual V, Allantaz F, Arce E, Punaro M, Banchereau J (2005) Role of interleukin-1 (IL-1) in the pathogenesis of systemic onset juvenile idiopathic arthritis and clinical response to IL-1 blockade. J Exp Med 201:1479-1486

74. Khaenam P, Rinchai D, Altman MC et al (2014) A transcriptomic reporter assay employing neutrophils to measure immunogenic activity of septic patients' plasma. J Transl Med 12:65

75. Jia S, Kaldunski M, Jailwala P et al (2011) Use of transcriptional signatures induced in lymphoid and myeloid cell lines as an inflammatory biomarker in type 1 diabetes. Physiol Genomics 43: 697-709

76. Jackson AM, Kanak MA, Grishman EK, Chaussabel D, Levy MF, Naziruddin B (2012) Gene expression changes in human islets exposed to type 1 diabetic serum. Islets 4:312-319

77. Cabrera SM, Henschel AM, Hessner MJ (2015) Innate inflammation in type 1 diabetes. Transl Res. doi:10.1016/j.trsl.2015.04.011

78. Chen YG, Cabrera SM, Jia S et al (2014) Molecular signatures differentiate immune states in type 1 diabetic families. Diabetes 63:3960-3973

79. Chen YG, Mordes JP, Blankenhorn EP et al (2013) Temporal induction of immunoregulatory processes coincides with agedependent resistance to viral-induced type 1 diabetes. Genes Immun 14:387-400

80. Levy H, Wang X, Kaldunski M et al (2012) Transcriptional signatures as a disease-specific and predictive inflammatory biomarker for type 1 diabetes. Genes Immun 13:593-604

81. Wang X, Jia S, Geoffrey R, Alemzadeh R, Ghosh S, Hessner MJ (2008) Identification of a molecular signature in human type 1 diabetes mellitus using serum and functional genomics. J Immunol 180:1929-1937

82. Aly TA, Ide A, Humphrey K et al (2005) Genetic prediction of autoimmunity: initial oligogenic prediction of anti-islet autoimmunity amongst DR3/DR4-DQ8 relatives of patients with type 1A diabetes. J Autoimmun 25(Suppl):40-45

83. Dogan Y, Akarsu S, Ustundag B, Yilmaz E, Gurgoze MK (2006) Serum IL-1 $\beta$, IL-2, and IL-6 in insulin-dependent diabetic children. Mediat Inflamm 2006:59206

84. Hussain MJ, Maher J, Warnock T, Vats A, Peakman M, Vergani D (1998) Cytokine overproduction in healthy first degree relatives of patients with IDDM. Diabetologia 41:343-349

85. Meyers AJ, Shah RR, Gottlieb PA, Zipris D (2010) Altered Tolllike receptor signaling pathways in human type 1 diabetes. J Mol Med (Berl) 88:1221-1231

86. Bradshaw EM, Raddassi K, Elyaman W et al (2009) Monocytes from patients with type 1 diabetes spontaneously secrete proinflammatory cytokines inducing Th17 cells. J Immunol 183: 4432-4439

87. Plesner A, Greenbaum CJ, Gaur LK, Ernst RK, Lernmark A (2002) Macrophages from high-risk HLA-DQB $1 * 0201 / * 0302$ type 1 diabetes mellitus patients are hypersensitive to lipopolysaccharide stimulation. Scand J Immunol 56:522-529

88. Kayserova J, Vcelakova J, Stechova K et al (2014) Decreased dendritic cell numbers but increased TLR9-mediated interferonalpha production in first degree relatives of type 1 diabetes patients. Clin Immunol 153:49-55

89. Zhang Q, Fillmore TL, Schepmoes AA et al (2013) Serum proteomics reveals systemic dysregulation of innate immunity in type 1 diabetes. J Exp Med 210:191-203 
90. von Herrath M, Sanda S, Herold K (2007) Type 1 diabetes as a relapsing-remitting disease? Nat Rev Immunol 7:988-994

91. Petrich de Marquesini LG, Fu J, Connor KJ et al (2010) IFN- $\gamma$ and IL-10 islet-antigen-specific T cell responses in autoantibodynegative first-degree relatives of patients with type 1 diabetes. Diabetologia 53:1451-1460

92. Pugliese A (2013) The multiple origins of type 1 diabetes. Diabet Med 30:135-146

93. Zhi W, Purohit S, Carey C, Wang M, She JX (2010) Proteomic technologies for the discovery of type 1 diabetes biomarkers. J Diabetes Sci Technol 4:993-1002

94. Moulder R, Bhosale SD, Erkkila T et al (2015) Serum proteomes distinguish children developing type 1 diabetes in a cohort with HLA-conferred susceptibility. Diabetes 64:2265-2278

95. Burch TC, Morris MA, Campbell-Thompson M, Pugliese A, Nadler JL, Nyalwidhe JO (2015) Proteomic analysis of disease stratified human pancreas tissue indicates unique signature of type 1 diabetes. PLoS One 10, e0135663

96. Simpson LJ, Ansel KM (2015) MicroRNA regulation of lymphocyte tolerance and autoimmunity. J Clin Invest 125:2242-2249

97. Nielsen LB, Wang C, Sorensen K et al (2012) Circulating levels of microRNA from children with newly diagnosed type 1 diabetes and healthy controls: evidence that miR-25 associates to residual beta-cell function and glycaemic control during disease progression. Exp Diabetes Res 2012:896362

98. Takahashi P, Xavier DJ, Evangelista AF et al (2014) MicroRNA expression profiling and functional annotation analysis of their targets in patients with type 1 diabetes mellitus. Gene 539:213223

99. Akirav EM, Lebastchi J, Galvan EM et al (2011) Detection of beta cell death in diabetes using differentially methylated circulating DNA. Proc Natl Acad Sci U S A 108:19018-19023

100. Fisher MM, Watkins RA, Blum J et al (2015) Elevations in circulating methylated and unmethylated preproinsulin DNA in newonset type 1 diabetes. Diabetes 64:3867-3872
101. Herold KC, Usmani-Brown S, Ghazi T et al (2015) beta cell death and dysfunction during type 1 diabetes development in at-risk individuals. J Clin Invest 125:1163-1173

102. Lebastchi J, Deng S, Lebastchi AH et al (2013) Immune therapy and beta-cell death in type 1 diabetes. Diabetes 62:1676-1680

103. von Herrath MG, Fujinami RS, Whitton JL (2003) Microorganisms and autoimmunity: making the barren field fertile? Nat Rev Microbiol 1:151-157

104. Chen Z, Barbi J, Bu S et al (2013) The ubiquitin ligase Stub1 negatively modulates regulatory $\mathrm{T}$ cell suppressive activity by promoting degradation of the transcription factor Foxp3. Immunity 39:272-285

105. van Loosdregt J, Fleskens V, Fu J et al (2013) Stabilization of the transcription factor Foxp3 by the deubiquitinase USP7 increases Treg-cell-suppressive capacity. Immunity 39:259-271

106. Kaldunski M, Jia S, Geoffrey R et al (2010) Identification of a serum-induced transcriptional signature associated with type 1 diabetes in the BioBreeding rat. Diabetes 59:2375-2385

107. Bottazzo GF, Florin-Christensen A, Doniach D (1974) Islet-cell antibodies in diabetes mellitus with autoimmune polyendocrine deficiencies. Lancet 2:1279-1283

108. Cabrera SM, Wang X, Chen YG et al (2015) Interleukin-1 antagonism moderates the inflammatory state associated with type 1 diabetes during clinical trials conducted at disease onset. Eur J Immunol DOI:10.1002/eji.201546005

109. Woo W, LaGasse JM, Zhou Z et al (2000) A novel highthroughput method for accurate, rapid, and economical measurement of multiple type 1 diabetes autoantibodies. J Immunol Methods 244:91-103

110. Miyara M, Yoshioka Y, Kitoh A et al (2009) Functional delineation and differentiation dynamics of human CD4+ T cells expressing the FoxP3 transcription factor. Immunity 30:899-911

111. Eisenbarth GS (1986) Type I diabetes mellitus. A chronic autoimmune disease. N Engl J Med 314:1360-1368

112. Coppieters KT, von Herrath MG (2014) The type 1 diabetes signature: hardwired to trigger inflammation? Diabetes 63:3581-3583 